\title{
Nasopharyngeal microbiota evaluation in three cohorts of children in the Romanian pediatric population
}

\author{
Anca Streinu-Cercel ${ }^{1,2^{*}}$, Oana Săndulescu ${ }^{1,2}$, loana Berciu ${ }^{1,2}$, Alina Cristina Neguț ${ }^{1,2}$, Mihai Săndulescu ${ }^{1,3}$, \\ Ruxandra Aursulesei ${ }^{1}$, Adrian Streinu-Cercel ${ }^{1,2}$ \\ From The 10th Edition of the Scientific Days of the National Institute for Infectious Diseases "Prof Dr Matei \\ Bals" \\ Bucharest, Romania. 15-17 October 2014
}

\section{Background}

The nasopharyngeal microbiota represents one of the key factors related to infectious diseases in children [1]. The infectious agents and their resistance patterns are main factors driving disease severity. Nasopharyngeal carriage is high in children, especially for Staphylococcus aureus $[2,3]$.

\section{Methods}

We performed a screening study for nasopharyngeal carriage of Staphylococcus spp. in immunocompetent children aged 7-10 years old, attending a community school in central Bucharest (group 1), and in two groups of immunosuppressed children: children with hemato-oncologic diseases (lymphoma/leukemia) admitted to the Fundeni Clinical Institute, Bucharest (ages 2-10 years, group 2), and institutionalized children with vertically transmitted HIV infection, from the National Institute for Infectious Diseases "Prof. Dr. Matei Balş", Bucharest (ages 1-10 years, group 3).

\section{Results}

We analyzed data from 139 pharyngeal swabs (35.3\% in group 1,56.1\% group 2 and $8.6 \%$ group 3 ), and 143 nasal swabs (37.1\% group 1, 54.5\% group 2 and $8.4 \%$ group 3). Pharyngeal cultures were positive for Staphylococcus spp. in $28.6 \%$ of children in group $1,11.5 \%$ in group $2(\mathrm{p}=0.00755$ vs. group 1$)$ and $16.7 \%$ in group $3(\mathrm{p}=0.20045$ vs. group 1$)$. Of the positive pharyngeal samples, $92.9 \%$ were S. aureus in group 1, $100 \%$ in group 2 and $100 \%$ in group 3.

Nasal cultures were positive for Staphylococcus spp. in $84.9 \%$ of children in group $1,48.7 \%$ group 2 ( $\mathrm{p}=0 \mathrm{vs.}$ group 1$)$ and $50.0 \%$ group $3(\mathrm{p}=0.00391$ vs. group 1$)$. Of the positive nasal samples, $62.2 \%$ were identified as $S$. aureus in group $1,94.6 \%$ in group $2(\mathrm{p}=0.00027$ vs. group 1$)$ and $66.7 \%$ in group 3.

\section{Conclusion}

Pharyngeal carriage of Staphylococcus strains was low, however when positive, most strains were $S$. aureus. Nasopharyngeal carriage was significantly higher in immunocompetent children from the community compared to immunodepressed children. When present, $S$. aureus had a higher prevalence compared to coagulase-negative staphylococci (CoNS), particularly in immunodepressed children.

\section{Acknowledgements \\ 1) POSDRU/159/1.5/S/137390. \\ 2) Carol Davila University of Medicine and Pharmacy, Young Researchers}

Projects Grant Competition, contract number 28.335/04.11.2013.

\section{Authors' details}

'Carol Davila University of Medicine and Pharmacy, Bucharest, Romania. ${ }^{2}$ National Institute for Infectious Diseases "Prof. Dr. Matei Balş", Bucharest, Romania. ${ }^{3}$ Dental Concept Studio, Bucharest, Romania. 
Published: 15 October 2014

\section{References}

1. Mimica MJ, Bádue-Pereira MF: Staphylococcus aureus colonization in Brazilian children. GERMS 2014, 4:22.

2. den Heijer $C D$, van Bijnen EM, Paget WJ, Pringle M, Goossens $H$, Bruggeman CA, Schellevis FG, Stobberingh EE, APRES Study Team: Prevalence and resistance of commensal Staphylococcus aureus, including meticillin-resistant $S$ aureus, in nine European countries: a cross-sectional study. Lancet Infect Dis 2013, 13:409-15.

3. Preoțescu LL, Streinu-Cercel O: Prevalence of nasal carriage of $S$ aureus in children. GERMS 2013, 3:49-51.

doi:10.1186/1471-2334-14-S7-021

Cite this article as: Streinu-Cercel et al:: Nasopharyngeal microbiota evaluation in three cohorts of children in the Romanian pediatric population. BMC Infectious Diseases 2014 14(Suppl 7):021.

\section{Submit your next manuscript to BioMed Central} and take full advantage of:

- Convenient online submission

- Thorough peer review

- No space constraints or color figure charges

- Immediate publication on acceptance

- Inclusion in PubMed, CAS, Scopus and Google Scholar

- Research which is freely available for redistribution

Submit your manuscript at www.biomedcentral.com/submit 